\title{
Computation of Modified Bessel Functions and Their Ratios*
}

\author{
By D. E. Amos
}

\begin{abstract}
An efficient algorithm for calculating ratios $r_{\nu}(x)=I_{\nu+1}(x) / I_{\nu}(x), \nu \geqq 0, x \geqq 0$, is presented. This algorithm in conjunction with the recursion relation for $r_{\nu}(x)$ gives an alternative to other recursive methods for $I_{\nu}(x)$ when approximations for low-order Bessel functions are available. Sharp bounds on $r_{\nu}(x)$ and $I_{\nu}(x)$ are also established in addition to some monotonicity properties of $r_{v}(x)$ and $r_{\nu}^{\prime}(x)$.
\end{abstract}

Introduction. Olver's uniform asymptotic expansion ([1], [11], [12]) in the index $\nu$,

$$
\begin{gathered}
I_{\nu}(x) \sim \frac{1}{(2 \pi \nu)^{1 / 2}} \frac{e^{\nu \eta}}{\left(1+z^{2}\right)^{1 / 4}} \sum_{k=0}^{\infty} \frac{u_{k}(t)}{\nu^{k}}, \quad \nu \rightarrow \infty, \\
z=x / \nu, \quad \eta=\left(1+z^{2}\right)^{1 / 2}+\ln \frac{z}{1+\left(1+z^{2}\right)^{1 / 2}}, \quad t=\frac{1}{\left(1+z^{2}\right)^{1 / 2}}, \\
u_{0}(t)=1, \quad u_{k+1}(t)=\frac{1}{2} t^{2}\left(1-t^{2}\right) u_{k}^{\prime}(t)+\frac{1}{8} \int_{0}^{t}\left(1-5 \tau^{2}\right) u_{k}(\tau) d \tau, \quad k \geqq 0,
\end{gathered}
$$

in conjunction with backward recursion on

$$
I_{v-1}(x)=\frac{2 v}{x} I_{\nu}(x)+I_{\nu+1}(x)
$$

makes the $I_{\nu}(x)$ Bessel function easily calculable for $\nu \geqq 0$ and $x \geqq 0$. Other approaches ([6], [7], [10]) use backward recursion on (1) directly or indirectly in the form

$$
r_{v-1}=\frac{1}{2 v / x+r_{v}}, \quad r_{v}=\frac{I_{v+1}(x)}{I_{v}(x)}
$$

to generate approximations for the ratios

$$
r_{\alpha+k}(x)=I_{\alpha+k+1}(x) / I_{\alpha+k}(x), \quad \alpha=\nu-[\nu], \quad k=0,1, \cdots,[\nu],
$$

where $[\nu]$ is the integer part of $\nu$ and $0 \leqq \nu-[\nu]<1$. The identity

$$
I_{\nu}(x)=I_{\alpha}(x) \prod_{k=1}^{[\nu]} r_{\nu-k}(x), \quad \alpha=\nu-[\nu]
$$

is then applied when $I_{\alpha}(x)$ is known. When $I_{\alpha}(x)$ is not explicitly known, a normalizing relation [6]

Received September 29, 1972, revised March 5, 1973.

AMS (MOS) subject classifications (1970). Primary 65D20, 33A40; Secondary 41-04.

Key words and phrases. Modified Bessel functions, recursive computation, ratios of Bessel functions, bounds on Bessel functions.

* This work was supported by the United States Atomic Energy Commission.

Copyright @ 1974, American Mathematical Society 


$$
I_{\alpha}(x)+2 \sum_{m=1}^{\infty} \frac{(\alpha+m) \Gamma(m+2 \alpha)}{m ! \Gamma(1+2 \alpha)} I_{\alpha+m}(x)=\frac{(x / 2)^{\alpha} e^{x}}{\Gamma(\alpha+1)}
$$

in the form

$$
I_{\alpha}(x)=\frac{(x / 2)^{\alpha} e^{x} / \Gamma(\alpha+1)}{1+2 \sum_{m=1}^{\infty} \frac{(\alpha+m) \Gamma(m+2 \alpha)}{m ! \Gamma(1+2 \alpha)} \prod_{k=1}^{[\alpha+m]} r_{\alpha+m-k}}
$$

is commonly used (Miller algorithm). However, each product in the denominator of (4) is monotone increasing in $x$. In the evaluation of (4), the number of terms of the series must therefore increase with increasing $x$ to satisfy a given error criterion. In contrast, Olver's expansion in reciprocal powers through $\nu^{-9}$ can be used for $\nu>20$ for relative errors [11] on the order of $10^{-12}$ or better.

The main result of this paper is an algorithm for rapid evaluation of ratios $r_{\nu}(x)$. This result for the index $\nu-1$, in conjunction with (2) and (3) for indices $\nu-2$ to $\alpha=\nu-[\nu]$, provides another way of calculating $I_{\nu}(x)$ Bessel functions when Chebyshev expansions or rapid approximations for low-order Bessel functions $I_{\alpha}(x)$ are available. One cannot proceed with increasing indices because (1) and (2) are numerically unstable with forward recursion.

Sequences are generated by means of $I_{\alpha+k+1}=r_{\alpha+k} I_{\alpha+k}, k=0,1,2, \cdots, N-1$, after the ratios $r_{c+k}$ have been calculated. On the other hand, if c ne crmputes $I_{\nu}$ from Olver's expansic $\mathrm{n}$, then $I_{\nu+1}=r_{\nu} I_{\nu}$, and (1) can be applied in a backward fashion for the sequence $I_{\alpha+k}$ or some lower-order Bessel function. Olver's expansion is also convenient from a programming point of view because the leading term can be tested for both over and underflow. Scaling by exp $(-x)$ can be incorporated easily by altering only the leading coefficient,

$$
e^{-x} e^{\nu \eta}=\exp \left\{\nu\left[\frac{1}{z+\left(1+z^{2}\right)^{1 / 2}}+\ln \frac{z}{1+\left(1+z^{2}\right)^{1 / 2}}\right]\right\}
$$

From the point of view of speed, however, a proper implementation might also include the power series for $I_{\nu}(x)$ when $(x / 2)^{2}<\nu+1$ (17 terms give relative errors better than $10^{-13}$ ).

Asymptotically correct bounds, which approximate $r_{\nu}(x)$ to maximum errors on the order of $1 \%$ for $\nu \geqq 2$, are also derived. Corresponding bounds on $I_{\nu}(x)$ become available through (3). [3] has applications in which direct ratios are needed. The development and convergence proof of the algorithm parallels that of [2] for the iterated coerror function.

Simple Bounds. We start with Bessel's equation

$$
y_{\nu}^{\prime \prime}+\frac{1}{x} y_{\nu}^{\prime}-\frac{\left(x^{2}+\nu^{2}\right)}{x^{2}} y_{\nu}=0, \quad \nu \geqq 0, x \geqq 0,
$$

for $y_{\nu}(x)=I_{\nu}(x)$ and write it in the form

$$
\left(x y_{v}^{\prime}\right)\left(x y_{\nu}^{\prime}\right)^{\prime}=\left(x^{2}+\nu^{2}\right) y_{\nu} y_{\nu}^{\prime}
$$

Integration by parts on the right followed by a division with $x^{2} y_{\nu}{ }^{2}(x)$ produces 


$$
Y_{\nu}^{2}=1+\frac{\nu^{2}}{x^{2}}-\frac{2}{x^{2} y_{\nu}^{2}(x)} \int_{0}^{x} z y_{\nu}^{2}(z) d z
$$

where

$$
Y_{\nu}(x) \equiv \frac{I_{\nu}^{\prime}(x)}{I_{\nu}(x)}=\frac{1}{2}\left[r_{\nu}(x)+\frac{1}{r_{\nu-1}(x)}\right]
$$

is deduced from the relation $I_{\nu}^{\prime}(x)=\frac{1}{2}\left[I_{v+1}(x)+I_{\nu-1}(x)\right]$. If we replace $r_{v-1}(x)$ in (7) by its equivalent in (2), we get

$$
Y_{\nu}(x)=r_{\nu}(x)+\nu / x .
$$

Now, (6) shows that

$$
r_{\nu}(x)+\frac{\nu}{x}=Y_{\nu}(x) \leqq\left(1+\nu^{2} / x^{2}\right)^{1 / 2}
$$

and

$$
r_{\nu}(x) \leqq-\frac{\nu}{x}+\left(1+\nu^{2} / x^{2}\right)^{1 / 2}=\frac{x}{\nu+\left(x^{2}+\nu^{2}\right)^{1 / 2}} .
$$

On the other hand, replacement of $r_{v}$ in (2) by this upper bound yields

$$
r_{\nu-1}=\frac{1}{2 \nu / x+r_{\nu}} \geqq \frac{1}{\nu / x+\left(1+\nu^{2} / x^{2}\right)^{1 / 2}}=\frac{x}{\nu+\left(x^{2}+\nu^{2}\right)^{1 / 2}} .
$$

These, with a shift of index, produce the bounds

$$
\begin{aligned}
0 \leqq \frac{x}{\nu+1+\left(x^{2}+(\nu+1)^{2}\right)^{1 / 2}} \leqq r_{\nu}(x) \leqq \frac{x}{\nu+\left(x^{2}+\nu^{2}\right)^{1 / 2}} & <1, \\
& x \geqq 0, \nu \geqq 0 .
\end{aligned}
$$

Another shift of index gives the monotonicity and separation properties

$$
r_{\nu+1}(x) \leqq \frac{x}{\nu+1+\left(x^{2}+(\nu+1)^{2}\right)^{1 / 2}} \leqq r_{\nu}(x), \quad x \geqq 0, \nu \geqq 0 .
$$

Iterative improvement yields a new upper bound

$$
\begin{aligned}
r_{\nu-1}= & \frac{1}{2 \nu / x+r_{\nu}} \leqq \frac{1}{2 \nu / x-(\nu+1) / x+\left(1+((\nu+1) / x)^{2}\right)^{1 / 2}} \\
& \leqq \frac{x}{\nu-1+\left(x^{2}+(\nu+1)^{2}\right)^{1 / 2}}
\end{aligned}
$$

or, with a shift of index,

$$
\begin{aligned}
\frac{x}{\nu+1+\left(x^{2}+(\nu+1)^{2}\right)^{1 / 2}} \leqq r_{\nu}(x) \leqq \frac{x}{\nu+\left(x^{2}+(\nu+2)^{2}\right)^{1 / 2}} \\
x \geqq 0, \nu \geqq 0 .
\end{aligned}
$$

Improved Bounds. Certain second-order differential equations can be converted to Ricatti equations by means of a simple substitution. The substitution for (5) is 


$$
Y_{\nu}(x)=y_{\nu}^{\prime} / y_{\nu}=I_{\nu}^{\prime}(x) / I_{\nu}(x)
$$

and (5) becomes

$$
Y_{\nu}^{\prime}=1+\nu^{2} / x^{2}-Y_{v} / x-Y_{v}^{2}, \quad x \geqq 0, \nu \geqq 0,
$$

or, in view of (8),

$$
r_{\nu}^{\prime}=1-(1+2 v) r_{\nu} / x-r_{\nu}^{2} .
$$

In order to make effective use of these relations, we derive some simple inequalities. We start with (5) and form

$$
\int_{0}^{x}\left[y_{v}\left(z y_{\nu+1}^{\prime}\right)^{\prime}-y_{v+1}\left(z y_{v}^{\prime}\right)^{\prime}\right] d z=(2 v+1) \int_{0}^{x} \frac{y_{v}(z) y_{\nu+1}(z)}{z} d z
$$

for indices $\nu$ and $\nu+1$. Green's theorem integrates the left side to yield

$$
\left.z\left[y_{\nu} y_{\nu+1}^{\prime}-y_{\nu+1} y_{\nu}^{\prime}\right]\right|_{0} ^{x}=(2 \nu+1) \int_{0}^{x} \frac{y_{\nu} y_{\nu+1}}{z} d z, \quad \nu \geqq 0,
$$

which produces

$$
Y_{\nu+1}(x)-Y_{\nu}(x)=\frac{2 \nu+1}{x y_{\nu}(x) y_{\nu+1}(x)} \int_{0}^{x} \frac{y_{\nu}(z) y_{\nu+1}(z)}{z} d z, \quad x>0 .
$$

This shows that

$$
Y_{\nu+1}>Y_{\nu} \text { for } x>0 .
$$

On the other hand, the logarithm of $r_{\nu}$ gives $\ln r_{\nu}=\ln I_{\nu+1}-\ln I_{\nu}$ and differentiation produces

$$
r_{\nu}^{\prime} / r_{\nu}=Y_{\nu+1}-Y_{\nu}=r_{\nu+1}-r_{\nu}+1 / x .
$$

Applying (10) and (13) to this relation, we get

$$
0<r_{\nu}^{\prime}(x)<r_{\nu}(x) / x, \quad x>0 .
$$

This shows that $r_{\nu}{ }^{\prime}$ must be positive, and (12) shows that this can only happen if $r_{\nu}$ lies between the roots of

$$
1-(2 \nu+1) t / x-t^{2}=0
$$

The larger root gives the upper bound in

$$
0 \leqq r_{\nu}(x) \leqq-\frac{\nu+\frac{1}{2}}{x}+\left(1+\frac{\left(\nu+\frac{1}{2}\right)^{2}}{x^{2}}\right)^{1 / 2}=\frac{x}{\nu+\frac{1}{2}+\left(x^{2}+\left(\nu+\frac{1}{2}\right)^{2}\right)^{1 / 2}} .
$$

Iterative improvement with (2) gives a lower bound

$$
\begin{aligned}
0 & \leqq \frac{x}{\nu+\frac{1}{2}+\left(x^{2}+\left(\nu+\frac{3}{2}\right)^{2}\right)^{1 / 2}} \leqq r_{\nu}(x) \\
& \leqq \frac{x}{\nu+\frac{1}{2}+\left(x^{2}+\left(\nu+\frac{1}{2}\right)^{2}\right)^{1 / 2}}<1, \quad x \geqq 0, \nu \geqq 0 .
\end{aligned}
$$

Further improvement helps, but the expressions become more complicated. These 
bounds together with (2) can be used numerically to generate pairs of bounds by backward recursion. The continued fraction developed from (2) leads to bounds which are rational function approximations to $r_{\nu}(x)$.

The upper bound in (16) is clearly an improvement over the upper bound of (9) of the previous section. Because the lower bounds in (9) and (16) were obtained in the same manner, one also expects the lower bound of (16) to be sharper than the lower bound of (9). However, the upper bound of (16) is only an improvement on the upper bound of $(11)$ when $(\nu+1)(2 \nu+3)<x^{2} / 4$.

If we apply (15) to (12), replacing $r_{\nu}$ by $x r_{\nu}{ }^{\prime}$, we get an improved bound on $r_{\nu}{ }^{\prime}(x)$,

$$
0<r_{\nu}^{\prime}(x)<\frac{1}{\nu+1+\left(x^{2}+(\nu+1)^{2}\right)^{1 / 2}}<\frac{r_{\nu}(x)}{x}, \quad x>0 .
$$

A result, which is analogous to one of the main results of [2],

$$
\frac{r_{\nu+1}}{r_{\nu}}-1=\frac{I_{\nu+2} I_{\nu}-I_{\nu+1}^{2}}{I_{\nu+1}^{2}}<0, \quad x \geqq 0,
$$

follows from the definition of $r_{\nu}$ and (10).

Bounds on $I_{\nu}(x)$. If we let $C_{\nu}$ and $D_{\nu}$ be lower and upper bounds of the preceding sections,

$$
C_{\nu}(x) \leqq r_{\nu}(x) \leqq D_{\nu}(x),
$$

$I_{\nu}(x)$ can be bounded in the form

$$
I_{\nu-[\nu]}(x) \prod_{k=1}^{[\nu]} C_{\nu-k}(x) \leqq I_{\nu}(x) \leqq I_{\nu-\{\nu]}(x) \prod_{k=1}^{[\nu]} D_{\nu-k}(x), \quad \nu \geqq 1 .
$$

[1, p. 362] displays some simple bounds while [8] gives rational function bounds for $I_{v}$ suitably normalized.

The following derivation adds bounds which are quite sharp and have correct asymptotic behavior in one or more of the directions $x \rightarrow 0, x \rightarrow \infty$ or $\nu \rightarrow \infty$. We start with (8) and the definition $Y_{\nu}(x)$ from (7),

$$
I_{\nu}^{\prime} / I_{\nu}=r_{\nu}(x)+\nu / x .
$$

This is integrated between $z$ and $x$ for

$$
\ln \left[I_{\nu}(x) / I_{\nu}(z)\right]=\int_{z}^{x} r_{\nu}(t) d t+\nu \ln (x / z)
$$

or

$$
I_{\nu}(x)=(x / z)^{\nu} I_{\nu}(z) \exp \left\{\int_{z}^{x} r_{\nu}(t) d t\right\}
$$

The bounds are obtained by replacing $r_{\nu}(t)$ by the upper and lower bounds of (16). The result is

$$
L(x, z, \nu) \leqq I_{\nu}(x) \leqq U(x, z, \nu),
$$


(18a)

$U(x, z, \nu)$

$L(x, z, \nu)$

$$
=\left(\frac{x}{z}\right)^{\nu} I_{\nu}(z) \exp \left\{\frac{x^{2}-z^{2}}{\left(x^{2}+b^{2}\right)^{1 / 2}+\left(z^{2}+b^{2}\right)^{1 / 2}}\right\}\left[\frac{D_{\nu}(x)}{x} \cdot \frac{z}{D_{\nu}(z)}\right]^{\nu+1 / 2}, \quad z \leqq x,
$$

$$
=\left(\frac{x}{z}\right)^{\nu} I_{\nu}(z) \exp \left\{\frac{x^{2}-z^{2}}{\left(x^{2}+a^{2}\right)^{1 / 2}+\left(z^{2}+a^{2}\right)^{1 / 2}}\right\}\left[\frac{C_{\nu}(x)}{x} \cdot \frac{z}{C_{\nu}(z)}\right]^{\nu+1 / 2}, \quad z \leqq x,
$$

$a=\nu+\frac{3}{2}, \quad b=\nu+\frac{1}{2}, \quad D_{\nu}(x)=\frac{x}{b+\left(x^{2}+b^{2}\right)^{1 / 2}}, \quad C_{\nu}(x)=\frac{x}{b+\left(x^{2}+a^{2}\right)^{1 / 2}}$

with $U$ and $L$ interchanged for $x<z$. If $z \rightarrow 0$, (17) and (18a) reduce to

$$
\begin{gathered}
I_{\nu}(x)=\frac{(x / 2)^{\nu}}{\Gamma(\nu+1)} \exp \left\{\int_{0}^{x} r_{\nu}(t) d t\right\} \\
U(x, 0, \nu)=\left(\frac{2}{x}\right)^{1 / 2} \frac{\left(\nu+\frac{1}{2}\right)^{\nu+1 / 2}}{\Gamma(\nu+1)} \exp \left\{x D_{\nu}(x)\right\}\left[D_{\nu}(x)\right]^{\nu+1 / 2} \\
L(x, 0, \nu)=\left(\frac{2}{x}\right)^{1 / 2} \frac{(\nu+1)^{\nu+1 / 2}}{\Gamma(\nu+1)} \exp \left\{x D_{\nu+1}(x)\right\}\left[C_{\nu}(x)\right]^{\nu+1 / 2}
\end{gathered}
$$

while for $z \rightarrow \infty,(18 \mathrm{a})$, with $I_{\nu}(z) \sim e^{z} /(2 \pi z)^{1 / 2}$, yields

$$
\begin{aligned}
& U(x, \infty, \nu)=\frac{\exp \left\{\left(x^{2}+\left(\nu+\frac{3}{2}\right)^{2}\right)^{1 / 2}\right\}}{(2 \pi x)^{1 / 2}}\left[C_{\nu}(x)\right]^{\nu+1 / 2}, \\
& L(x, \infty, \nu)=\frac{\exp \left\{\left(x^{2}+\left(\nu+\frac{1}{2}\right)^{2}\right)^{1 / 2}\right\}}{(2 \pi x)^{1 / 2}}\left[D_{\nu}(x)\right]^{\nu+1 / 2} .
\end{aligned}
$$

By proper choices of $a$ and $b$ in (18a), the bounds corresponding to (11) can also be obtained. Another form which leads to an infinite product is derived by integrating (14)

$$
\ln \left[r_{\nu}(x) / r_{\nu}(z)\right]=\int_{z}^{x} r_{\nu+1}(t) d t-\int_{z}^{x} r_{\nu}(t) d t+\ln (x / z)
$$

and recurring to obtain

$$
\int_{z}^{x} r_{\nu}(t) d t=\sum_{k=0}^{N} \ln \left[\frac{x}{r_{\nu+k}(x)} \cdot \frac{r_{\nu+k}(z)}{z}\right]+\int_{z}^{x} r_{\nu+N+1}(t) d t, \quad N \geqq 0 .
$$

Then, this integral in (17) gives

$$
I_{\nu}(x)=\left(\frac{x}{z}\right)^{\nu} I_{\nu}(z) \prod_{k=0}^{N}\left[\frac{x}{r_{\nu+k}(x)} \cdot \frac{r_{\nu+k}(z)}{z}\right] \exp \left\{\int_{z}^{x} r_{\nu+N+1}(t) d t\right\} .
$$

The exponential term can be taken from (18a) with $\nu$ replaced by $\nu+N+1$ for upper and lower bounds which are arbitrarily close if $N$ is large enough and $r_{v+k}$ is computed according to the procedure outlined in the Introduction.

Computation of $r_{\nu}(x)$ and $I_{\nu}(x)$. The motivation for the computational procedure follows by manipulating (2) into the form

$$
\left[\left(r_{\nu+1} / r_{\nu}\right) r_{\nu}+2(\nu+1) / x\right] r_{\nu}=1
$$

and solving the quadratic equation for $r_{\nu}$, 


$$
r_{\nu}=\frac{x}{\nu+1+\left(R_{\nu+1} x^{2}+(\nu+1)^{2}\right)^{1 / 2}}, \quad R_{\nu+1}=\frac{r_{\nu+1}}{r_{\nu}} .
$$

Here we use the lower bound of (16) for an initial approximation,

$$
\begin{aligned}
& r_{\nu+k}^{0}=\frac{x}{\nu+k+\frac{1}{2}+\left(\left(\nu+k+\frac{3}{2}\right)^{2}+x^{2}\right)^{1 / 2}}, \\
& \qquad k=0,1,2, \cdots, N, N \geqq 1,
\end{aligned}
$$

and form iterates from (19),

$$
\begin{aligned}
& R_{\nu+k+1}^{m}=r_{\nu+k+1}^{m} / r_{\nu+k}^{m} \\
& \left.r_{\nu+k}^{m+1}=\frac{x}{\nu+k+1+\left((\nu+k+1)^{2}+x^{2} R_{\nu+k+1}^{m}\right)^{1 / 2}}\right\} \\
& k=0,1,2, \cdots, N-m-1, \quad m=0,1, \cdots, N-1 .
\end{aligned}
$$

The iteration diagram is shown in Fig. 1.

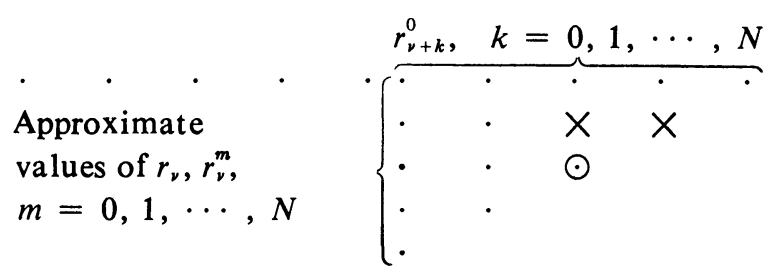

FiguRe 1

$r_{\nu}{ }^{m}$ is the approximate value of $r_{\nu}$. Since an iteration is a backward sweep along the lower diagonal, only two successive diagonals need to be stored during computation.

The convergence proof below shows that the columns of Fig. 1 converge to the appropriate quantity as $N \rightarrow \infty$. The algorithm can be terminated by comparing the entries $r_{\nu+1}{ }^{N-1}$ and $r_{v}{ }^{N}$ by means of the formulae

$$
d_{\nu}^{N}=\frac{1}{2(\nu+1) / x+r_{\nu+1}^{N-1}}, \quad \frac{\left|d_{\nu}^{N}-r_{\nu}^{N}\right|}{r_{\nu}^{N}}<\epsilon
$$

for an approximate relative error $\epsilon$. That is, $d_{\nu}{ }^{N}$ would be $r_{\nu}{ }^{N}$ if $r_{\nu+1}{ }^{N-1}$ were exact. In cases where $r_{\nu}{ }^{N}$ and $r_{\nu+1}{ }^{N-1}$ increase monotonically as $N \rightarrow \infty, d_{\nu}{ }^{N}$ is an upper bound on $r_{\nu}, r_{\nu}{ }^{N}$ is a lower bound, and $\epsilon$ is a rigorous relative error. The convergence for $\nu<10$ with values of $x$ in the range 1-20 was slow but for $\nu \geqq 10, N$ was 4 or less for at least 8 significant digits. It is therefore recommended that for $\nu<10$ the index be increased by an integer $K$ so that $\nu+K \geqq 10$, and $r_{\nu+K}$ be generated. (2) can then be used to get values for indices less than 10 .

To calculate $I_{\nu}(x)$, we generate $r_{\nu-1}(x)$, recur backward with (2) and evaluate (3) when $I_{\nu-\{\nu\}}(x)$ is available. References [4], [5] and [9] contain Chebyshev expansions of high accuracy for $\nu-[\nu]=0, \frac{1}{4}, \frac{1}{3}, \frac{1}{2}, \frac{2}{3}, \frac{3}{4}, 1$. Results of modest accuracy are also given in [5] for $-1 \leqq \nu \leqq 1$ and $x \leqq 8$. The asymptotic expansion for $I_{\nu}(x)$ suffices for corresponding accuracy for $-1 \leqq \nu \leqq 1$ and $x>8$. Olver's expansion as described in the Introduction can also be used to generate $I_{\nu}(x)$.

With more recent machines, the computation of a square root is on the order of three divide times. Each iteration of (20b) therefore results in only a few microseconds. 
Since computer multiplications require significantly more time to execute than additions or subtractions, squaring of the indexed quantity $(\nu \pm k+1)$ can be reduced to additions and subtractions by means of

$$
\begin{aligned}
y_{0} & =(\nu+1)^{2}, \quad u_{0}=\nu+1, \\
y_{k+1} & =y_{k} \pm u_{k} \pm u_{k}+1, \\
u_{k+1} & =u_{k} \pm 1,
\end{aligned} \quad k=0,1,2, \cdots,
$$

where $y_{k}=(\nu \pm k+1)^{2}$ and $u_{k}=\nu \pm k+1$. The plus sign is used for $r_{\nu+k}{ }^{0}$ along the first row of Fig. 1, while the minus sign is used during the backward sweep along the diagonal of Fig. 1.

For the numerical experiments, the algorithm was compared with backward recursion utilizing (2). For example, $r_{10}(100)$ was calculated from (2) with $r_{10+k}(100)=$ $0, r_{10+k}(100)=$ upper bound of (16), and $r_{10+k}(100)=$ lower bound of (16). For a relative error $\epsilon=5 \times 10^{-9}, k$ had to be at least 36,28 and 26 respectively. The algorithm met the error requirement at $N=6(6$ applications of (20a) and 15 applications of (20b)) producing 10 significant digits. The corresponding computation for $r_{10}(500)$ produced the numbers $k=90,62,62$ and $N=4$ respectively. Other experiments repeated this trend where the differences between the two methods became increasingly apparent as $x$ exceeded $2 \nu$. The experiments also showed the methods to be comparable in terms of the number of equivalent multiplications or divisions when $x$ was small compared with $\nu$. The backward recursive approach was superior for intermediate values of $x$ and $10 \leqq \nu \leqq 70$. However, the algorithm performed uniformly better for $\nu>70$, giving $N \leqq 4$ when $\epsilon=5 \times 10^{-9}$. The comparisons above utilized the optimal value of $k$ for a given $(\epsilon, x)$ pair. In practice, an algorithm constructed from (2) may not perform as well since the optimal value of $k$ in $r_{v+k}(x)$ is not known a priori without a parameter study. The algorithm, while terminated in a nonrigorous fashion, always produced the accuracy requested. Rigorous termination is possible by utilizing the upper and lower bounds constructed in Theorem 2 below.

Convergence of the Algorithm. We first show in Theorem 1 that an algorithm formed from (20b) with $r_{\nu+k}{ }^{0}$ given by the lower bound of (11) converges as $N \rightarrow \infty$. Theorem 2, which contains the corresponding results for (20a) and (20b), follows because the initial approximation $r_{\nu+k}{ }^{0}$ is sharper than that of Theorem 1 .

THEOREM 1. If $x \geqq 0$ and $\nu \geqq 0$, the sequences $r_{\nu+k}{ }^{m}$ generated by (20b) with

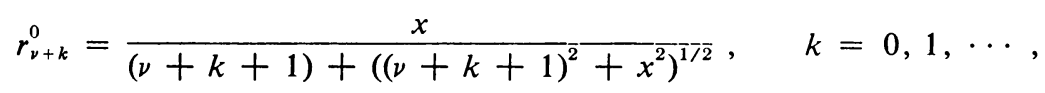

converges to $r_{v+k}$ as $m \rightarrow \infty$ for each $k \geqq 0$.

For the proof, we write (19) and (20b) in the form

$$
\begin{aligned}
& r_{\nu+k}=\frac{1}{\frac{\nu+k+1}{x}+\left(\left(\frac{\nu+k+1}{x}\right)^{2}+\frac{r_{\nu+k+1}}{r_{\nu+k}}\right)^{1 / 2}}, \\
& r_{\nu+k}^{m+1}=\frac{1}{\frac{\nu+k+1}{x}+\left(\left(\frac{\nu+k+1}{x}\right)^{2}+\frac{r_{\nu+k+1}^{m}}{r_{\nu+k}^{m}}\right)^{1 / 2}}
\end{aligned}
$$


and consider $x>0$ since $r_{\nu+k}(0)=r_{\nu+k}^{0}(0)=0$. The proof consists of constructing sequences of upper and lower bounds on $r_{v+k}{ }^{m}$ which converge to $r_{v+k}$. Let

$$
C_{k}^{0}=r_{\nu+k}^{0}=\frac{1}{\frac{\nu+k+1}{x}+\left(\left(\frac{\nu+k+1}{x}\right)^{2}+1\right)^{1 / 2}} .
$$

Then, since $C_{k}{ }^{0}$ is a lower bound, (2), with a shift of index, yields an upper bound

$$
D_{k}^{0}=\frac{1}{2(\nu+k+1) / x+C_{k+1}^{0}}
$$

on $r_{v+k}$. Then,

$$
D_{k}^{0}>r_{\nu+k}>r_{\nu+k}^{0}=C_{k}^{0} .
$$

Substitution of these bounds into the expressions $r_{v+k+1} / r_{v+k}$ and $r_{v+k+1} / r_{v+k}{ }^{0}$ yields

$$
\frac{C_{k+1}^{0}}{D_{k}^{0}}<\frac{r_{\nu+k+1}}{r_{\nu+k}}<\frac{D_{k+1}^{0}}{C_{k}^{0}} \text { and } \frac{C_{k+1}^{0}}{D_{k}^{0}}<\frac{r_{v+k+1}^{0}}{r_{\nu+k}^{0}}<\frac{D_{k+1}^{0}}{C_{k}^{0}} \text {. }
$$

These bounds can be used in (21) to replace $r_{v+k+1} / r_{v+k}$ and $r_{v+k+1}{ }^{0} / r_{v+k}{ }^{0}$ under the square root. Then, for $m=0, D_{k}{ }^{1}>r_{v+k}>C_{k}{ }^{1}$ and $D_{k}{ }^{1}>r_{v+k}{ }^{1}>C_{k}{ }^{1}$ where

$$
\begin{aligned}
& D_{k}^{1}=\frac{1}{\frac{\nu+k+1}{x}+\left(\left(\frac{\nu+k+1}{x}\right)^{2}+\frac{C_{k+1}^{0}}{D_{k}^{0}}\right)^{1 / 2}} \text { and } \\
& C_{k}^{1}=\frac{1}{\frac{\nu+k+1}{x}+\left(\left(\frac{\nu+k+1}{x}\right)^{2}+\frac{D_{k+1}^{0}}{C_{k}^{0}}\right)^{1 / 2}} .
\end{aligned}
$$

Continuing in this way, we can inductively construct sequences of bounds $D_{k}{ }^{m}$ and $C_{k}{ }^{m}$ on $r_{k}$ and $r_{k}{ }^{m}$. However, convergence is obtained by showing monotonicity of each sequence. Thus, for $m=0$ we need to show that

$$
C_{k}^{1} \geqq C_{k}^{0} \quad \text { and } \quad D_{k}^{0} \geqq D_{k}^{1} .
$$

$C_{k}{ }^{1}>C_{k}{ }^{0}$ follows from the result

$$
\begin{aligned}
\frac{D_{k+1}^{0}}{C_{k}^{0}} & =\frac{\frac{\nu+k+1}{x}+\left(\left(\frac{\nu+k+1}{x}\right)^{2}+1\right)^{1 / 2}}{\frac{2(\nu+k+2)}{x}+C_{k+2}^{0}} \\
& =\frac{\frac{\nu+k+1}{x}+\left(\left(\frac{\nu+k+1}{x}\right)^{2}+1\right)^{1 / 2}}{\frac{2(\nu+k+2)}{x}-\frac{\nu+k+3}{x}+\left(\left(\frac{\nu+k+3}{x}\right)^{2}+1\right)^{1 / 2}} \\
& =\frac{\frac{\nu+k+1}{x}+\left(\left(\frac{\nu+k+1}{x}\right)^{2}+1\right)^{1 / 2}}{\frac{\nu+k+1}{x}+\left(\left(\frac{\nu+k+3}{x}\right)^{2}+1\right)^{1 / 2}}<1,
\end{aligned}
$$


since $D_{k+1}{ }^{0} / C_{k}^{0}$ replaces 1 under the square root in the expression for $C_{k}^{0}=r_{p+k}{ }^{0}$. For $D_{k}{ }^{0} \geqq D_{k}{ }^{1}$ we have

$$
\begin{aligned}
D_{k}^{1} & =\frac{1}{\frac{\nu+k+1}{x}+\left(\left(\frac{\nu+k+1}{x}\right)^{2}+C_{k+1}^{0}\left(C_{k+1}^{0}+\frac{2(\nu+k+1)}{x}\right)\right)^{1 / 2}} \\
& =\frac{1}{2(\nu+k+1) / x+C_{k+1}^{0}}=D_{k}^{0} .
\end{aligned}
$$

To summarize for $m=0$, we have

$$
D_{k}^{0}=D_{k}^{1}>r_{\nu+k}>C_{k}^{1}>C_{k}^{0} \text { and } D_{k}^{0}=D_{k}^{1}>r_{\nu+k}^{1}>C_{k}^{1}>C_{k}^{0} \text {. }
$$

Now we repeat the induction steps between (24) and (25) for $m=1$. Thus, (28) applied to $r_{v+k+1} / r_{v+k}$ and $r_{v+k+1}{ }^{1} / r_{v+k}{ }^{1}$ yields

$$
\frac{C_{k+1}^{1}}{D_{k}^{1}}<\frac{r_{v+k+1}}{r_{v+k}}<\frac{D_{k+1}^{1}}{C_{k}^{1}} \text { and } \frac{C_{k+1}^{1}}{D_{k}^{1}}<\frac{r_{v+k+1}^{1}}{r_{v+k}^{1}}<\frac{D_{k+1}^{1}}{C_{k}^{1}} \text {. }
$$

These expressions with (21) give new bounds $D_{k}{ }^{2}$ and $C_{k}{ }^{2}$,

$$
D_{k}^{2}>r_{\nu+k}>C_{k}^{2} \text { and } D_{k}^{2}>r_{\nu+k}^{2}>C_{k}^{2}
$$

where

$$
D_{k}^{2}=\frac{1}{\frac{\nu+k+1}{x}+\left(\left(\frac{\nu+k+1}{x}\right)^{2}+\frac{C_{k+1}^{1}}{D_{k}^{1}}\right)^{1 / 2}}
$$

and

$$
C_{k}^{2}=\frac{1}{\frac{\nu+k+1}{x}+\left(\left(\frac{\nu+k+1}{x}\right)^{2}+\frac{D_{k+1}^{1}}{C_{k}^{1}}\right)^{1 / 2}} .
$$

But (28) shows

$$
\frac{C_{k+1}^{1}}{D_{k}^{1}}>\frac{C_{k+1}^{0}}{D_{k}^{0}} \quad \text { and } \quad \frac{D_{k+1}^{1}}{C_{k}^{1}}<\frac{D_{k+1}^{0}}{C_{k}^{0}}
$$

and it follows that

$$
C_{k}^{2}>C_{k}^{1} \quad \text { and } \quad D_{k}^{2}<D_{k}^{1}
$$

Thus, for $m=1$ we have

$$
D_{k}^{0}=D_{k}^{1}>D_{k}^{2}>r_{\nu+k}>C_{k}^{2}>C_{k}^{1}>C_{k}^{0}
$$

and

$$
D_{k}^{0}=D_{k}^{1}>D_{k}^{2}>r_{\nu+k}^{2}>C_{k}^{2}>C_{k}^{1}>C_{k}^{0}
$$

for $x>0$.

Continuing in this way, we compute inductively a sequence $D_{k}{ }^{m}$ which is bounded and monotone decreasing, and a sequence $C_{k}{ }^{m}$ which is bounded and monotone increasing with $r_{v+k}$ and $r_{v+k}{ }^{m}$ between these bounds. Each sequence therefore has a 
limit $D_{k}$ and $C_{k}$ such that

$$
\begin{aligned}
& D_{k}=\frac{1}{\frac{\nu+k+1}{x}+\left(\left(\frac{\nu+k+1}{x}\right)^{2}+\frac{C_{k+1}}{D_{k}}\right)^{1 / 2}}, \\
& C_{k}=\frac{1}{\frac{\nu+k+1}{x}+\left(\left(\frac{\nu+k+1}{x}\right)^{2}+\frac{D_{k+1}}{C_{k}}\right)^{1 / 2}} .
\end{aligned}
$$

Solving for each of these square roots and squaring gives

$$
D_{k}=\frac{1}{2(\nu+k+1) / x+C_{k+1}}, \quad C_{k}=\frac{1}{2(\nu+k+1) / x+D_{k+1}} .
$$

Elimination between these expressions yields

$$
\begin{aligned}
& D_{k}=\frac{1}{2(\nu+k+1) / x+\frac{1}{2(\nu+k+2) / x+D_{k+2}}}, \\
& C_{k}=\frac{1}{2(\nu+k+1) / x+\frac{1}{2(\nu+k+2) / x+C_{k+2}}} .
\end{aligned}
$$

Each of these leads to the continued fraction for $r_{\nu+k}$ which can be developed similarly by repeated application of (2). Therefore, $D_{k}=C_{k}=r_{v+k}$ and $r_{v+k}{ }^{m}$ converges to $r_{v+k}$.

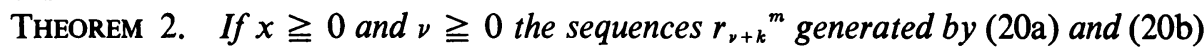
converge to $r_{v+k}$ as $m \rightarrow \infty$ for each $k \geqq 0$.

Again we observe that $r_{v+k}(0)=r_{\nu+k}{ }^{0}(0)=0$ and consider $x>0$. In the proof of this theorem, we construct bounds $\bar{C}_{k}{ }^{m}$ and $\bar{D}_{k}{ }^{m}$ in a fashion analogous to those of Theorem 1 and compare these bounds with those of Theorem 1 . We first note that $\bar{C}_{k}{ }^{0}=r_{v+k}{ }^{0}$ of (20a) is a better approximation to $r_{v+k}$ than $C_{k}{ }^{0}$ of Theorem 1 . That is, $r_{\nu+k}>\bar{C}_{k}{ }^{0}>C_{k}{ }^{0}$ for $x>0$. We also have from (2), with a shift of index, an upper bound $\bar{D}_{k}^{0}$,

$$
D_{k}^{0}=\frac{1}{2(\nu+k+1) / x+\bar{C}_{k+1}^{0}}<\frac{1}{2(\nu+k+1) / x+C_{k+1}^{0}}=D_{k}^{0},
$$

on $r_{v+k}$. Thus, for $m=0$ we have

$$
C_{k}^{0}<\bar{C}_{k}^{0}=r_{\nu+k}^{0}<r_{\nu+k}<D_{k}^{0}<D_{k}^{0} .
$$

It follows therefore that

$$
\begin{aligned}
& \frac{C_{k+1}^{0}}{D_{k}^{0}}<\frac{\bar{C}_{k+1}^{0}}{\bar{D}_{k}^{0}}<\frac{r_{\nu+k+1}}{r_{\nu+k}}<\frac{\bar{D}_{k+1}^{0}}{\bar{C}_{k}^{0}}<\frac{D_{k+1}^{0}}{C_{k}^{0}}, \\
& \frac{C_{k+1}^{0}}{D_{k}^{0}}<\frac{\bar{C}_{k+1}^{0}}{\bar{D}_{k}^{0}}<\frac{r_{\nu+k+1}^{0}}{r_{\nu+k}^{0}}<\frac{D_{k+1}^{0}}{\bar{C}_{k}^{0}}<\frac{D_{k+1}^{0}}{C_{k}^{0}} .
\end{aligned}
$$

We define 


$$
\begin{aligned}
& \bar{C}_{k}^{1}=\frac{1}{\frac{\nu+k+1}{x}+\left(\left(\frac{\nu+k+1}{x}\right)^{2}+\frac{\bar{D}_{k+1}^{0}}{\bar{C}_{k}^{0}}\right)^{1 / 2}} \text { and } \\
& \bar{D}_{k}^{1}=\frac{1}{\frac{\nu+k+1}{x}+\left(\left(\frac{\nu+k+1}{x}\right)^{2}+\frac{\bar{C}_{k+1}^{0}}{\bar{D}_{k}^{0}}\right)^{1 / 2}} .
\end{aligned}
$$

The fact that these are bounds on $r_{\nu+k}$ and $r_{\nu+k}{ }^{1}$ follows from (21) and (30). Then, comparing the expressions in (21), (30), (31), and (25) for $m=0$, we have

$$
\begin{aligned}
& C_{k}^{1}<\bar{C}_{k}^{1}<r_{\nu+k}<\bar{D}_{k}^{1}<D_{k}^{1}, \\
& C_{k}^{1}<\bar{C}_{k}^{1}<r_{\nu+k}^{1}<\bar{D}_{k}^{1}<D_{k}^{1} .
\end{aligned}
$$

Now, $r_{v+k}$ and $r_{v+k}{ }^{1}$ are in the same relative positions with respect to the $C$ 's and $D$ 's for $m=1$ as $r_{v+k}$ and $r_{v+k}^{0}$ were for $m=0$ in (29). We therefore inductively compute

$$
\begin{aligned}
& C_{k}^{m}<\bar{C}_{k}^{m}<r_{\nu+k}<D_{k}^{m}<D_{k}^{m}, \quad m=1,2, \cdots . \\
& C_{k}^{m}<\bar{C}_{k}^{m}<r_{\nu+k}^{m}<D_{k}^{m}<D_{k}^{m},
\end{aligned}
$$

But in Theorem 1 it was shown that both $C_{k}{ }^{m}$ and $D_{k}{ }^{m}$ converge to $r_{v+k}$. Therefore $r_{v+k}{ }^{m}$ as well as $\bar{C}_{k}{ }^{m}$ and $\bar{D}_{k}{ }^{m}$ converge to $r_{v+k}$ as $m \rightarrow \infty$.

Because the initial approximation to $r_{v+k}$ is sharper in Theorem 2 than that of Theorem 1, one expects fewer iterations for a given accuracy. Numerical experiments show this to be the case.

Notice that the proof of Theorem 2 implies that an algorithm based on (20b) and started with any lower bound which is an improvement over (11) (e.g., (16)) converges to $r_{v+k}$. Hence

THEOREM 3. If $r_{\nu+k} \geqq r_{\nu+k}^{0} \geqq x /\left(\nu+k+1+\left((\nu+k+1)^{2}+x^{2}\right)^{1 / 2}\right), x \geqq 0$ and $\nu \geqq 0$, then the sequences generated by (20b) converge to $r_{\nu+k}$ as $m \rightarrow \infty$ for each $k \geqq 0$.

Acknowledgement. The author wishes to acknowledge the assistance of Mrs. Sharon L. Daniel for her part in coding the numerical experiments for a CDC 6600 computer.

Sandia Laboratories

Numerical Analysis Division 5122

Albuquerque, New Mexico 87115

1. M. Abramowitz \& I. A. Stegun (Editors), Handbook of Mathematical Functions, With Formulas, Graphs and Mathematical Tables, Nat. Bur. Standards Appl. Math. Ser., 55, Superintendent of Documents, U.S. Government Printing Office, Washington, D.C., 1964. MR 29 \#4914.

2. D. E. Amos, "Bounds on iterated coerror functions and their ratios," Math. Comp., v. 27,1973 , pp. $413-427$.

3. D. E. Amos, Evaluation of Some Cumulative Distribution Functions by Numerical Quadrature, Symposium Proceedings, Sixth Annual Symposium of Interface: Computer Science and Statistics, University of California, Berkeley, October 16-17, 1972.

4. C. W. Clenshaw, Chebyshev Series for Mathematical Functions, National Physical Laboratory Mathematical Tables, vol. 5, Department of Scientific and Industrial Research, Her Majesty's Stationery Office, London, 1962. MR 26 \#362. 
5. C. W. Clenshaw \& S. M. Picken, Chebyshev Series for Bessel Functions of Fractional Order, National Physical Laboratory Mathematical Tables, vol. 8, Her Majesty's Stationery Office, London, 1966. MR 34 \#2948.

6. W. GAUTSCHI, "Computational aspects of three-term recurrence relations," SIAM Rev., v. 9, 1967, pp. 24-82. MR 35 \#3927.

7. Y. L. LUKE, On Generating Bessel Functions by Use of the Backward Recurrence Formula, Aerospace Research Laboratories Report ARL 72-0030, Wright-Patterson Air Force Base, Ohio.

8. Y. L. LUKE, "Inequalities for generalized hypergeometric functions," J. Approximation Theory, v. 5, 1972, pp. 41-65.

9. Y. L. Luke, The Special Functions and Their Approximations. Vol. II, Math. in Sci. and Engineering, vol. 53, Academic Press, New York, 1969. MR 40 \#2909.

10. F. W. J. OlVER, "Numerical solution of second order linear difference equations," J. Res. Nat. Bur. Standards Sect. B, v. 71B, 1967, pp. 111-129. MR 36 \#4841.

11. F. W. J. Olver, Tables for Bessel Functions of Moderate or Large Orders, National Physical Laboratory Mathematical Tables, vol. 6, Department of Scientific and Industrial Research, Her Majesty's Stationery Office, London, 1962. MR 26 \# 5190.

12. F. W. J. Olver, "The asymptotic expansion of Bessel functions of large order," Philos. Trans. Roy Soc. London Ser. A, v. 247, 1954, pp. 328-368. MR 16, 696. 